Revue d'histoire de l'Amérique française

REYUE D.HISTOIRE DE L'AMÉRIQUE FRANÇAISE

COOK, Janet L., Coalescence of Styles. The Ethnic Heritage of St. John River Valley Regional Furniture, 1763-1851 (Montréal, McGill-Queen's University Press, 2001), xxii-304 p.

\title{
Paul-Louis Martin
}

Volume 55, numéro 4, printemps 2002

URI : https://id.erudit.org/iderudit/010445ar

DOI : https://doi.org/10.7202/010445ar

Aller au sommaire du numéro

Éditeur(s)

Institut d'histoire de l'Amérique française

ISSN

0035-2357 (imprimé)

1492-1383 (numérique)

Découvrir la revue

Citer ce compte rendu

Martin, P.-L. (2002). Compte rendu de [COOK, Janet L., Coalescence of Styles. The Ethnic Heritage of St. John River Valley Regional Furniture, 1763-1851 (Montréal, McGill-Queen's University Press, 2001), xxii-304 p.] Revue d'histoire de l'Amérique française, 55(4), 609-613. https://doi.org/10.7202/010445ar d'utilisation que vous pouvez consulter en ligne.

https://apropos.erudit.org/fr/usagers/politique-dutilisation/ 
Cet ouvrage s'avère néanmoins une excellente synthèse des connaissances actuelles en histoire de l'alimentation en Nouvelle-France. Il saura intéresser aussi bien le profane que l'historien chevronné, ainsi que les ethnologues. Il est à espérer que cet ouvrage pique la curiosité de jeunes chercheurs et les pousse à investiguer l'un ou l'autre champ de cette captivante facette de notre histoire.

CATHERINE FERLAND

CÉLAT

Université Laval

COOK, Janet L., Coalescence of Styles. The Ethnic Heritage of St. John River Valley Regional Furniture, I763-I85 I (Montréal, McGill-Queen's University Press, 200 I), xxii-304 p.

L'étude des paysages humanisés et des habitations, des objets domestiques et des artefacts contribue depuis longtemps à lever le voile sur les anonymes de l'histoire, à mieux connaître les premiers occupants d'un territoire, leurs rapports entre eux et avec les nouveaux arrivants. Si les archéologues ont développé des techniques éprouvées pour analyser les traces de cette culture matérielle, les muséologues et les historiens de l'art ne sont pas en reste : en approfondissant davantage les contextes de production et d'utilisation des objets et des collections muséologiques, ils viennent ajouter quelques pierres à la construction d'une histoire culturelle, plus crédible parce que moins univoque, plus difficile à saisir aussi puisque fondée sur une dynamique d'échanges, d'emprunts et d'adaptations.

Janet L. Cook livre ici le résultat d'une enquête fouillée sur le mobilier et le genre de vie des occupants d'une région naturelle du NouveauBrunswick, la vallée du fleuve Saint-Jean. À l’arrivée des Européens, des groupes de Malécites et de Micmacs occupent l'ensemble de ce bassin versant. Dans les années 1760 naîtra, à l'embouchure du fleuve, la ville de Saint John, fondée par des migrants du Massachussetts. Au nord, la vallée du Haut-Saint-Jean accueillera d'abord des Acadiens, à compter de 1785 et, peu après, quelques familles de Canadiens français provenant de la Côtedu-Sud. Les deux zones recevront par la suite des apports ethniques de sources diverses, américaine au nord, écossaise et irlandaise au sud. Deux territoires d'étude donc, celui du sud, doté d'un pôle urbain et d'un port maritime, celui du nord, essentiellement rural, agricole et forestier.

La période choisie par l'auteure, 1763-1851, présente l'avantage de scruter les premiers moments de la domestication des deux espaces, à peine 
transformés jusque-là par la présence continue des Amérindiens. Elle peut y suivre la progression de l'occupation humaine, vérifier les impacts de l'arrivée des nouveaux venus et observer les processus d'échanges, d'adaptation ou d'adoption d'usages, de techniques et de formes nouvelles. Malgré son unité géographique, la vallée du Saint-Jean offre à cette époque l'image de deux milieux sociaux bien distincts : le premier est une porte ouverte sur le monde, l'autre est un îlot relativement clos, presque un isolat social. On pourrait s'interroger sur le bien-fondé d'une telle approche qui offre peu d'éléments socio-économiques comparables, sauf que l'auteure entend situer précisément son analyse dans l'ordre culturel des échanges. Elle limite son étude aux meubles meublants, de repos, de rangement, de consommation, etc., créés sur place par les habitants et les artisans parce qu'ils sont, d'après elle, issus directement du milieu, contrairement à d'autres objets domestiques, en céramique, en verre, en métal ou en fibres qui proviennent de l'extérieur de la vallée et qui, à son avis, se prêtent beaucoup moins à l'analyse culturelle comparée. Il s'agit d'un point de vue difficile à partager : les choix de vaisselle, d'ustensiles et d'accessoires domestiques révèlent, tout autant que les meubles, la pénétration d'usages, de pratiques alimentaires, voire le mimétisme social et les influences culturelles à l'œuvre dans un milieu donné. Mais passons.

Après avoir exposé en introduction sa méthode de travail, Janet L. Cook consacre un chapitre d'une trentaine de pages aux pionniers de la partie nord du fleuve Saint-Jean, cette vallée encore connue de nos jours sous le nom de Madawaska. Utilisant surtout des sources indirectes, de nombreux témoignages de voyageurs et quelques rapports d'arpenteurs et d'observateurs de passage, Janet $\mathrm{L}$. Cook parvient à esquisser un tableau à première vue réaliste de l'établissement des familles pionnières acadiennes et canadiennes-françaises. Elle relève bon nombre des caractères propres à la domestication initiale des paysages sauvages : petite taille des habitations, emploi de matériaux naturels bruts et peu transformés (couvrement d'écorces, cheminées de terre, etc.), frugalité du régime alimentaire, simplicité et rudesse du genre de vie. La pauvreté des arrivants, leur outillage limité, l'éloignement des marchés et, conséquemment, le coût élevé de marchandises aussi banales que les clous, les carreaux de vitres, les poêles à chauffage et les outils de métier contraignent les quelque cinquante familles de défricheurs à vivre au quotidien dans un cadre plutôt fruste. Pendant combien de temps? Dix ans, vingt ans? L'auteure est muette sur le sujet, faute de disposer de sources notariées et de données qui lui auraient permis de suivre de plus près les univers domestiques, de situer 
le seuil d'une existence un peu plus douce, en somme de cerner plus exactement les échanges culturels.

Passant rapidement outre la dynamique des établissements domestiques, l'auteure mène plutôt son analyse comparée à partir d'un choix de meubles créés trente ou quarante ans après les premiers établissements, soit entre 1825 et 1845 . Cette sélection de meubles, au surplus très limitée, sept chaises droites, deux berçantes, deux tables, un coffre, une armoire et une commode, témoigne d'emprunts stylistiques et formels, sans pour autant fournir l'indice d'une acculturation généralisée : il faudrait plus que des éléments de décor sur quelques chaises, une parenté structurale ou encore l'interprétation simplifiée du style Empire d'une seule commode pour qualifier l'importance des échanges culturels. En fait, plusieurs des pièces retenues sont en tout point identiques à celles qu'on retrouve ailleurs dans l'est et dans le milieu d'origine des colons : les chaises à siège d'assemblage et les coffres à faces unies sont communs sur la Côte-du-Sud. La démonstration est d'autant moins évidente que l'auteure semble méconnaître certains faits : les sièges des chaises paysannes du Québec n'étaient pas tous tressés en babiche, loin de là, et il se trouve partout dans l'est quantité de meubles de facture simple, des pièces essentiellement utilitaires, qui sont aux antipodes des spécimens élaborés illustrés dans le répertoire très sélectif et très "régime français » de Jean Palardy. Janet L. Cook puise en effet ses références et ses comparaisons dans cette «bible», écritelle, pourtant publiée il y a presque quarante ans. Elle oublie du même coup nombre d'ajustements apportés depuis lors à la connaissance des univers matériels de l'époque. Autre exemple d'assertion dépassée, cette fois à propos de l'architecture au Régime français : l'emploi des bardeaux de cèdre et de pin, comme revêtement de toit ou de pignon, est bel et bien connu des Français qui les nomment «essentes» dans l'est de leur pays. Contrairement à l'opinion de Palardy, endossée par l'auteur, on a utilisé le bardeau sur les toits des maisons très tôt au XviI ${ }^{\mathrm{e}}$ siècle, sans attendre l'influence américaine. Par contre, comme la pose de ce matériau exige beaucoup de clous, faits à la main et coûteux, les colons en voie d'établissement lui ont préféré un couvrement de planches, quitte à adopter le bardeau plus tard sur leur grande maison, mieux construite et plus ornée. Dans l'ensemble, donc, on sent l'auteure moins à l'aise dans l'interprétation et l'analyse du cadre de vie des colons du Haut-Saint-Jean. L'absence de sources premières, le petit nombre de spécimens de meubles examinés et surtout une grille de lecture incomplète et dépassée rendent sa démonstration peu convaincante. 
À l'opposé, beaucoup mieux documentés et plus probants apparaissent les deux chapitres consacrés au sud de la vallée et principalement à la ville de Saint John. Aux premiers migrants anglo-américains de 1763 s'ajoutent des Loyalistes quelques années plus tard, puis des immigrants écossais vers 1815, suivis par des Irlandais dont le nombre va s'accroître dans les années 1840. L'auteure dispose cette fois de sources variées pour mener ses analyses : meubles de tous types, objets bien documentés et en nombre suffisant, inventaires de biens, témoignages d'époque, annonces de journal, recensements, listes d'artisans, etc., si bien que sa démonstration des échanges techniques et formels gagne en crédibilité. La diversité sociale y est évidemment plus grande que dans la vallée supérieure du fleuve, la ville portuaire accueillant dès sa naissance des administrateurs coloniaux, des navigateurs, des marchands, des artisans, des pêcheurs, des agriculteurs et toute la faune des navires au long cours, en fait l'éventail complet d'une cité reliée au reste du monde. Cela se perçoit dans la variété, la richesse, voire l'exotisme des goûts et des façons de vivre : les meubles de style et de bois précieux y sont plus nombreux et des ateliers de meubliers et de menuisiers ne tardent pas à y prospérer. L'espace nous manque pour détailler le soin que l'auteure met à comparer les spécimens métropolitains aux versions locales, à retracer l'usage des nouveaux bois, à décrire savamment les formes simplifiées ou la grammaire décorative. Ce sont ainsi les artisans écossais qui introduisent à Saint John des interprétations plus mesurées et moins exubérantes des styles à la mode dans la première moitié du $\mathrm{XIX}^{\mathrm{e}}$ siècle, donnant ainsi une couleur locale distinctive aux intérieurs domestiques. Lorsque les Irlandais arrivent, fuyant les grandes famines du milieu du siècle, les formes et les techniques industrielles ont déjà commencé à supplanter les produits de l'artisanat. Tout en répétant quelques formes familières, certains de ces artisans deviennent plutôt manufacturiers de chaises et entreprennent de satisfaire le goût d'une clientèle américaine sans cesse croissante. L'auteure termine donc son étude au moment où se déploie une industrie qui vient répondre tout autrement aux besoins des gens.

La méthode d'analyse de Janet L. Cook reste fort intéressante, malgré quelques faiblesses évoquées précédemment. On se plaît à penser qu'une approche aussi bien fondée et une analyse aussi fine pourrait conduire à renouveler la connaissance du mobilier dans certaines régions et villes du Québec. À la condition toutefois de pouvoir retracer dans les musées et dans les collections privées des spécimens bien documentés et à la provenance assurée, ce qui n'est pas une mince tâche. Quant à l'identité parti- 
culière de cette région du Nouveau-Brunswick, comme beaucoup d'autres en Amérique, elle résulte d'une sédimentation des cultures autant que de leur interpénétration occasionnelle, mais pour en saisir toute l'essence, la profondeur et le rythme, il faudrait ajouter à ce profil préliminaire bien d'autres dimensions de la culture matérielle. L’ouvrage demeure néanmoins une épure de belle qualité.

PAUL-LOUIS MARTIN

Département de sciences humaines Université du Québec à Trois-Rivières

COURTEMANCHE, Andrée et Martin PÂQUET, dir., Prendre la route : l'expérience migratoire en Europe et en Amérique du Nord du XIVe au XXe siècle (Hull, Éditions Vents d'Ouest, 200I), $243 p$.

Ce livre, qui est le fruit d'un colloque tenu à Moncton en 1999 sur les stratégies socioculturelles des migrations, aborde un sujet un peu moins vaste que ne le suggère le titre. Puisque le colloque a réuni des chercheurs français, belges et canadiens, le volet européen ne concerne que les migrants français ou belges, tandis que les articles sur l'Amérique du Nord, à une exception près, se concentrent sur les immigrants ou les émigrants canadiens. C'est la notion de l'expérience migratoire qui relie l'analyse des mouvements des deux côtés de l'Atlantique et rend de la cohérence à la démarche comparative. Ce concept permet de voir le migrant comme acteur autonome dont l'horizon d'attente pourtant s'insère à l'intérieur d'un champ de force. L'approche est donc à la fois subjectiviste et systémique.

Le livre est divisé en quatre parties qui se recoupent partiellement. La première, intitulée «l'expérience de la rencontre», souligne la nature paradoxale de la migration, qui oscille entre les deux pôles de solidarité et d'altérité, de maintien et de rupture. Le premier article, par Yves Roby, est une étude subtile de la transformation de l'identité canadienne-française en Nouvelle-Angleterre pendant les années 1880. Cette époque voit apparaître chez l'élite immigrante sensible aux critiques nativistes un nouveau discours qui se calque sur celui de la survivance - celui de l'Américain modèle. Ces deux discours coexistent, tout en étant fort différents, voire opposés, d'où l'émergence d'une identité à trait d'union. C'est la naissance du Franco-Américain. Dans l'article qui suit, Jean Morency considère la représentation ambivalente de l'expérience migratoire dans l'œuvre de Gabrielle Roy. 